\title{
Gastro- or Duodenojejunostomy Leaks After Pancreatoduodenectomy: Single Center Experience and Narrative Literature Review
}

\author{
Knut Jørgen Labori $^{1,2}\left(D \cdot\right.$ Tore Tholfsen $^{1} \cdot$ Sheraz Yaqub $^{1,2} \cdot$ Kristoffer Lassen $^{1,3} \cdot$ Dyre Kleive $^{1} \cdot$ Anne Waage $^{1}$
}

Received: 17 April 2021 / Accepted: 25 May 2021 / Published online: 15 June 2021

(C) 2021 The Author(s)

\begin{abstract}
Background and Methods Gastro- or duodenojejunostomy leaks after pancreatoduodenectomy is rare. This study aims to analyze the incidence, management, and outcome of gastro- or duodenojejunostomy leaks after pancreatoduodenectomy based on a single center experience from 2004 to 2020 with a narrative literature review.

Results Of a total of 1494 pancreatoduodenectomies, eight patients with gastrojejunostomy $(\mathrm{n}=1)$ or duodenojejunostomy $(\mathrm{n}=7$ ) leak were identified from the institutional pancreatic database. All leaks were treated operatively. In two patients dismantling of the duodenojejunostomy, distal gastrectomy, and closure of the pyloric and jejunal side, a percutaneous endoscopic gastrostomy and a feeding jejunostomy ultimately had to be performed after an unsuccessful attempt of gastrojejunostomy and suture of the duodenojejunostomy, respectively. The literature search revealed three more studies specifically addressing this complication after pancreatoduodenectomy (36 patients of a total of 4739 pancreatoduodenectomies). Based on an analysis of the current study and the literature review, the overall incidence of gastro- or duodenojejunostomy leaks after pancreatoduodenectomy was $0.71 \%$ (44/6233 pancreatoduodenectomies). The occurrence of a gastro- or duodenojejunostomy leak was associated with a concomitant postoperative pancreatic fistula in $50 \%$ of the cases, an increased length of hospital stay, and a mortality rate of $15.9 \%$. Surgical treatment was performed in $84 \%$ of the cases.

Conclusion Gastro- or duodenojejunostomy leak is a rare complication after pancreatoduodenectomy. Prompt diagnosis and early repair is important. In most cases, a surgical intervention is necessary for a good outcome. Under salvage conditions, a bailout strategy may be to temporarily dismantle the gastro- or duodenojejunal anastomosis.
\end{abstract}

Keywords : Pancreatoduodenectomy · Gastroenterostomy · Duodenoenterostomy · Leakage

\section{Introduction}

A gastrojejunostomy $(\mathrm{GJ})$ is part of the reconstruction in many surgical procedures. Classic pancreatoduodenectomy (cPD) with distal gastrectomy and GJ and pylorus-preserving pancreatoduodenectomy (PPPD) with duodenojejunostomy (DJ) are considered equally effective for the treatment of

Knut Jørgen Labori

uxknab@ ous-hf.no

1 Department of Hepato-Pancreato-Biliary Surgery, Oslo University Hospital, Rikshospitalet, Oslo, Norway

2 Institute of Clinical Medicine, University of Oslo, Oslo, Norway

3 Institute of Clinical Medicine, Arctic University of Norway, Tromsø, Norway pancreatic and periampullary tumors $[1,2]$. Anastomotic leakage of the GJ or DJ after PD is rare and can sometimes be severe and difficult to manage. There are limited publications focusing on this potentially serious complication after PD [1, 3-5]. This study aims to analyze the incidence, management, and outcome of GJ or DJ leaks after PD based on single center experience and a literature review.

\section{Material and Methods}

From 2004 to 2020, eight patients with GJ or DJ leak were identified from the institutional pancreatic database of 1494 PDs. Patients were scored as having GJ or DJ leakage when a defect at this anastomosis was encountered at reoperation or when there was sufficient radiologic evidence of leakage [4]. PD was performed as previously described [6, 7]. From 2004 
to 2011, pancreatic surgery in Oslo was performed at two different hospitals: Ullevål University Hospital and Rikshospitalet. In October 2011, the departments merged into the high-volume HPB surgical center it is today, with all procedures being performed at Rikshospitalet. From 2004 to 2011, Ullevål University Hospital had PPPD as standard procedure, whereas cPD procedure was preferred at Rikshospitalet. PPPD has been the standard approach since 2012. However, a distal gastrectomy is performed when there is a question of ischemia or tumor involvement of the proximal duodenum. Antecolic GJ or DJ was performed approximately $40 \mathrm{~cm}$ distal to the hepaticojejunostomy by single running continuous monofilament 4-0 suture. A surgical drain was placed in all patients. The nasogastric tube was removed immediately after skin closure and before endotracheal extubation [7]. There were no dietary restrictions after surgery, but patients were encouraged to begin carefully and increase intake according to tolerance over postoperative day (POD) 1-4. As a general rule, well-nourished patients not achieving adequate energy/protein requirement by oral intake within 5 days after the surgery received artificial nutritional support. Malnourished patients and those who developed severe postoperative complications early after operation received early supplementary artificial nutrition.

Clinical presentation, radiologic findings, treatment, and outcome of patients with GJ or DJ leakage were analyzed. Comprehensive complication index (CCI) and alternative fistula risk score (aFRS) were measured by means of the online tools (https://www.assessurgery.com/about_cci-calcula- tor/) (https://www.evidencio.com/models/show/621). Postoperative pancreatic fistulas (POPF), postpancreatectomy hemorrhage, and bile leaks were defined and graded according to international definitions [8-10]. The hospital review board approved the study (19/04710) according to the general guidelines provided by the regional ethics committee. Continuous variables are presented as a median (range).

A PubMed/MEDLINE literature search was conducted using the search terms alone and in combination of "pancreatoduodenectomy" OR "pancreaticoduodenectomy" AND "gastrojejunostomy" OR "duodenojejunostomy" OR "gastroenterostomy" OR "duodenoenterostomy" AND "leakage" OR "fistulas." The search period ended as of 31 December 2020. Reference lists of all included papers and related articles were screened manually to identify missed but relevant studies. The final inclusion of papers to cite and reference was made at the discretion of the authors.

\section{Results}

Eight patients (male $\mathrm{n}=5$, median age 63 years) with a GJ or DJ leak were identified in the series of 1494 PDs, resulting in

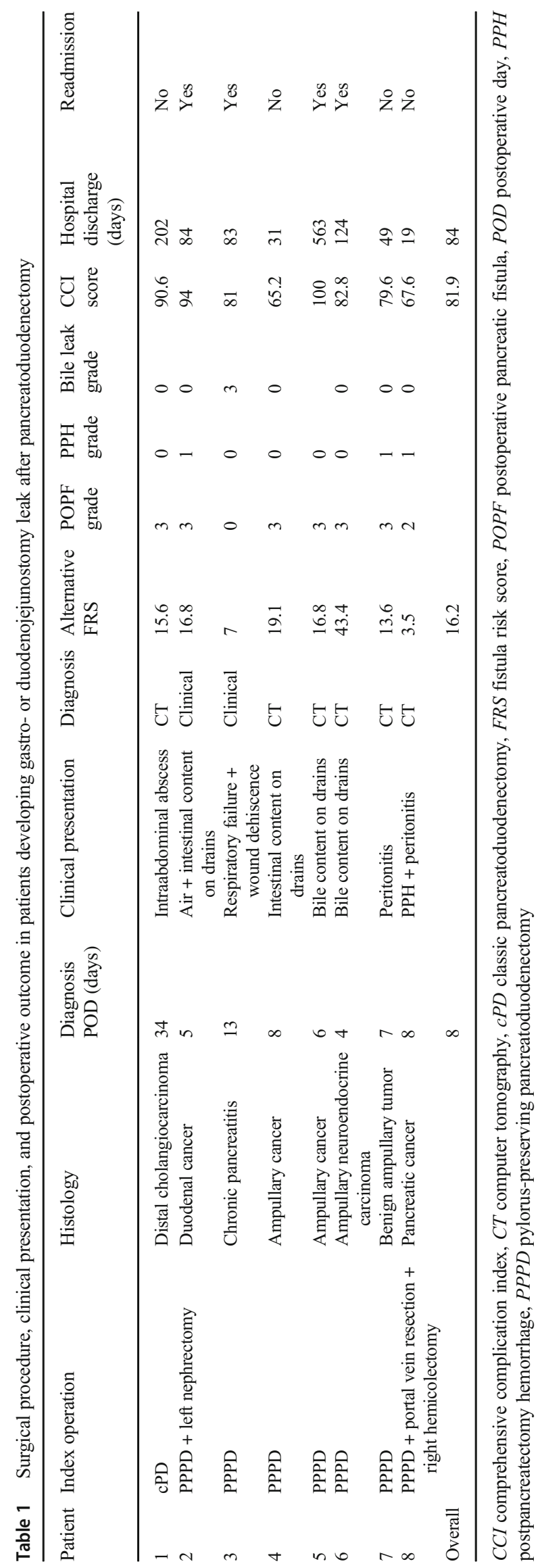


an incidence of $0.54 \%$. Details on surgical procedure, histological diagnosis, clinical presentation, and the postoperative course are presented in Table 1. Seven patients underwent PPPD, and in one patient, a cPD was performed. Six patients had a concomitant POPF grade $\mathrm{C}$ and one patient a POPF grade B. Median CCI was 81.9, and length of hospital stay was 84 days. Ninety-day mortality was zero. One patient died in hospital 18 months after a PPPD from complications related to the initial DJ leak diagnosed on POD 6. He had undergone extensive radiation therapy to the abdomen 4 decades previously.

The leaks presented as intestinal fluid, bile, or air on the surgical drains in four patients. In the remaining four patients, the leak presented as intraabdominal abscess, peritonitis, wound dehiscence/respiratory failure, or postpancreatectomy hemorrhage, respectively. The leaks were diagnosed on median POD 8 (range 4-34). All cases underwent surgery as definitive treatment of the leak. In six patients, there was a high grade of clinical and radiological suspicion of a GJ or DJ
Table 2 Surgical treatment of patients developing gastro- or duodenojejunostomy leak after pancreatoduodenectomy

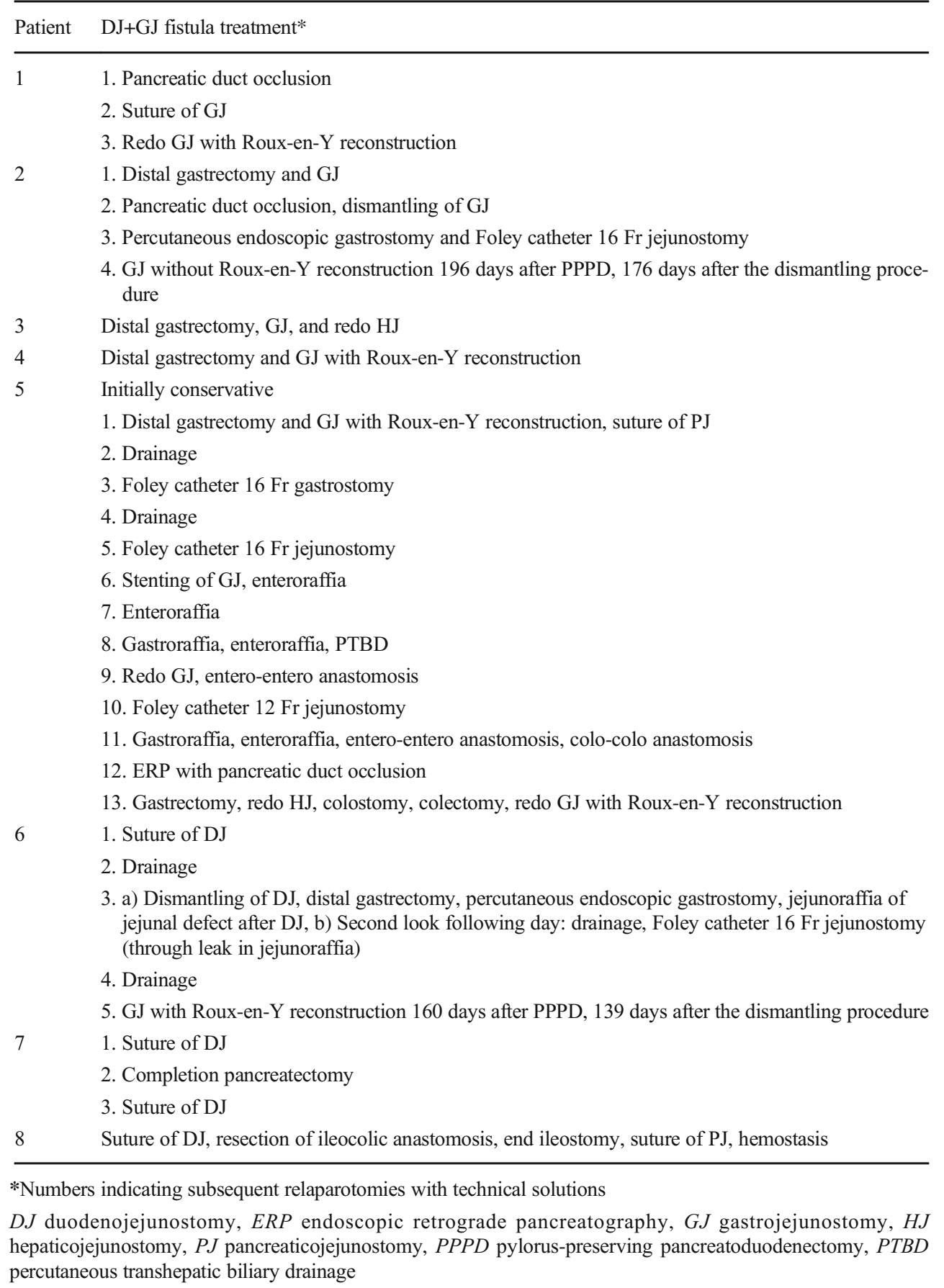


leak before relaparotomy. In one patient, an initial conservative approach with percutaneous drains was chosen, but the patient finally underwent a relaparotomy eight days after the diagnosis of a DJ leak.

Table 2 displays the surgical management of the eight patients. Suture of the DJ defect was performed successfully in two patients. Following this procedure, one of these patients had two reoperations: first a completion pancreatectomy followed by yet another suture of the DJ defect. In one patient, a glue injection into the pancreatic duct was performed due to POPF grade C. Following this procedure, the patient had two reoperations: first a suture of the GJ defect followed by a redo GJ with Roux-en-Y reconstruction. Two patients had a reoperation with redo GJ, and one of the patients also underwent a redo hepaticojejunostomy; both patients had a successful outcome. Three patients had a particularly complicated course. One patient eventually died after 18 months of hospitalization due to complications related to the DJ leak, POPF, enteral leak from the blind end of the pancreatobiliary jejunal limb, intestinal obstruction, and massive intestinal adhesions. In two patients, dismantling of the GJ and DJ was performed after an unsuccessful GJ without Roux-en-Y reconstruction and suture of the DJ defect, respectively (Table 2, Fig. 1). After the dismantling procedure, a percutaneous endoscopic gastrostomy (PEG, Mic Key®) catheter was inserted for drainage of the stomach, in addition to broad drainage of the concomitant POPF. Both patients had a 16 Fr Foley catheter securely placed into the efferent jejunal limb for enteral nutrition (Fig. 1). These two patients finally recovered, and a reoperation with a GJ with and without Roux-en-Y reconstruction was successfully performed 139 and 176 days after the dismantling procedure, respectively.

The literature search revealed three studies including 36 patients developing a GJ/DJ leak after a total of 4739 PDs (Table 3). Including the current case series, the overall incidence of GJ/DJ leak after PD was $0.71 \%$. GJ/DJ leaks were associated with concomitant POPF in $50 \%$ of the cases, increased length of hospital stay, and a mortality rate of $15.9 \%$.

\section{Discussion}

The current case series and literature review found an incidence of GJ/DJ leak after PD of $0.71 \%$. The occurrence of a GJ/DJ leak was associated with a concomitant POPF in $50 \%$ of the cases, an increased length of hospital stay, and a mortality rate of $15.9 \%$. Surgical treatment was performed in $84 \%$ of the cases, and all studies emphasized the importance of prompt diagnosis and surgery as the preferred treatment approach.

Three studies evaluated risk factors for GJ/DJ leak after PD [3-5]. Winter et al. found that perioperative risk factors included a preoperative blood urea nitrogen-to-creatinine ratio $>20$ (odds ratio $=6 ; \mathrm{p}=0.01$ ) and intraoperative blood loss $\geq 1 \mathrm{~L}$

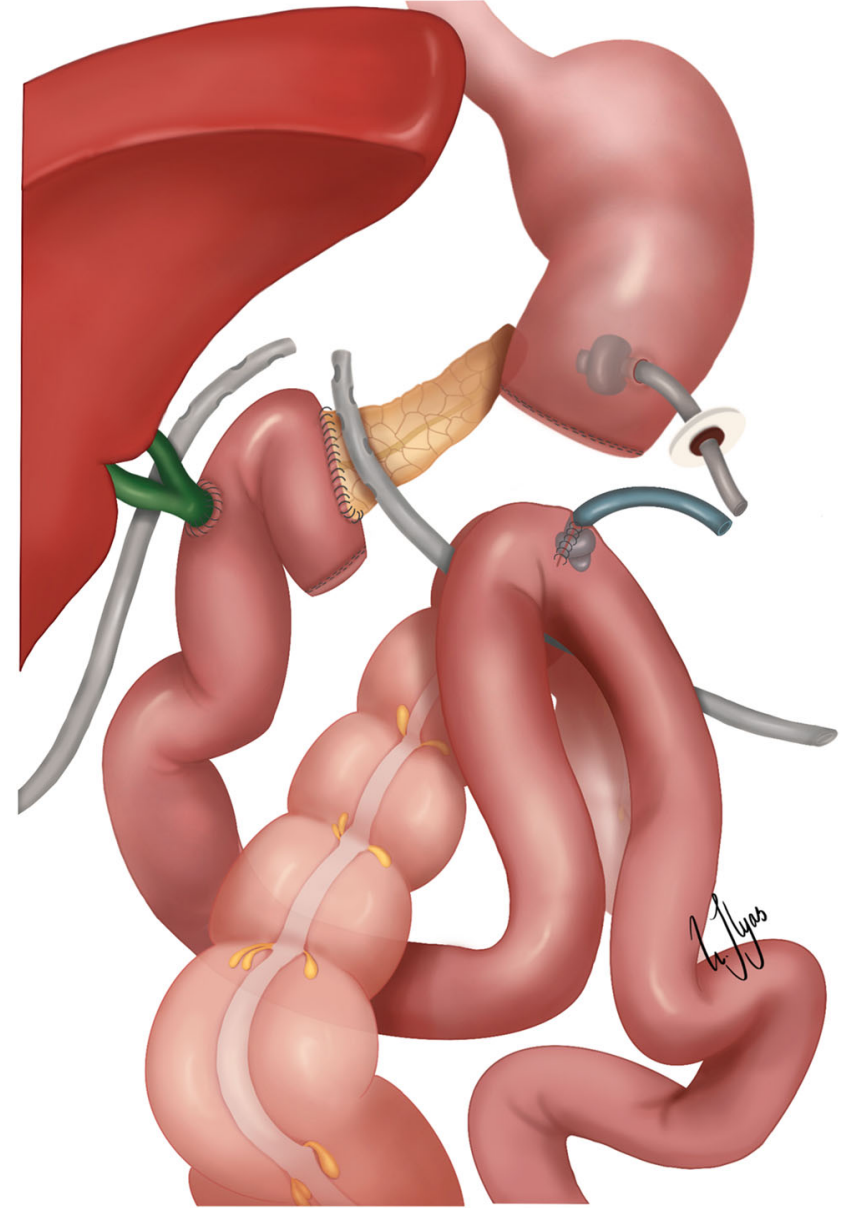

Fig. 1 Bailout strategy used under salvage conditions in two cases of duodenojejunostomy leaks after pancreatoduodenectomy. After dismantling of the gastro- or duodenojejunostomy, distal gastrectomy, and closure of the pyloric and jejunal side, a percutaneous gastrostomy (PEG, Mic Key®) was applied with aid of a gastroscope, and the gastric serosal wall around the catheter was secured to the anterior abdominal wall. Enteral access for nutrition was fashioned with a 16 Fr Foley catheter through the closed jejunal defect in the first patient and $5 \mathrm{~cm}$ distal of the jejunal defect in the second patient. The jejunal serosal wall around the enteral catheter was firmly sutured to the anterior abdominal wall in a wide area around the catheter. Drains were placed for drainage of a concomitant pancreatic fistula. After healing of the concomitant fistulas, resolution of inflammatory changes, and restitution of nutrition, a gastrojejunostomy was safely performed after about 6 months

(odds ratio $=6 ; \mathrm{p}=0.03$ ) in a multivariate model [3]. In a casecontrol study, Mazza et al. found that cases developing GJ/DJ leak showed lower preoperative serum hemoglobin $(\mathrm{p}=0.021)$ and increased preoperative radiotherapy $(p=0.037)$ [5]. Moreover, these patients experienced a more demanding intraoperative course including an increased estimated blood loss (median 600 vs. $400 \mathrm{~mL}, \mathrm{p}=0.002$ ), a higher rate of blood transfusion ( $31 \%$ vs. $8 \%$; $\mathrm{p}=0.047$ ), and a longer operative time (median 360 vs. $318 \mathrm{~min}$; $=0.038$ ). Eshuis et al. found that an additional organ resection during the index procedure was significantly more frequently performed and that the operation time was significantly longer (366 vs $301 \mathrm{~min}$; p 


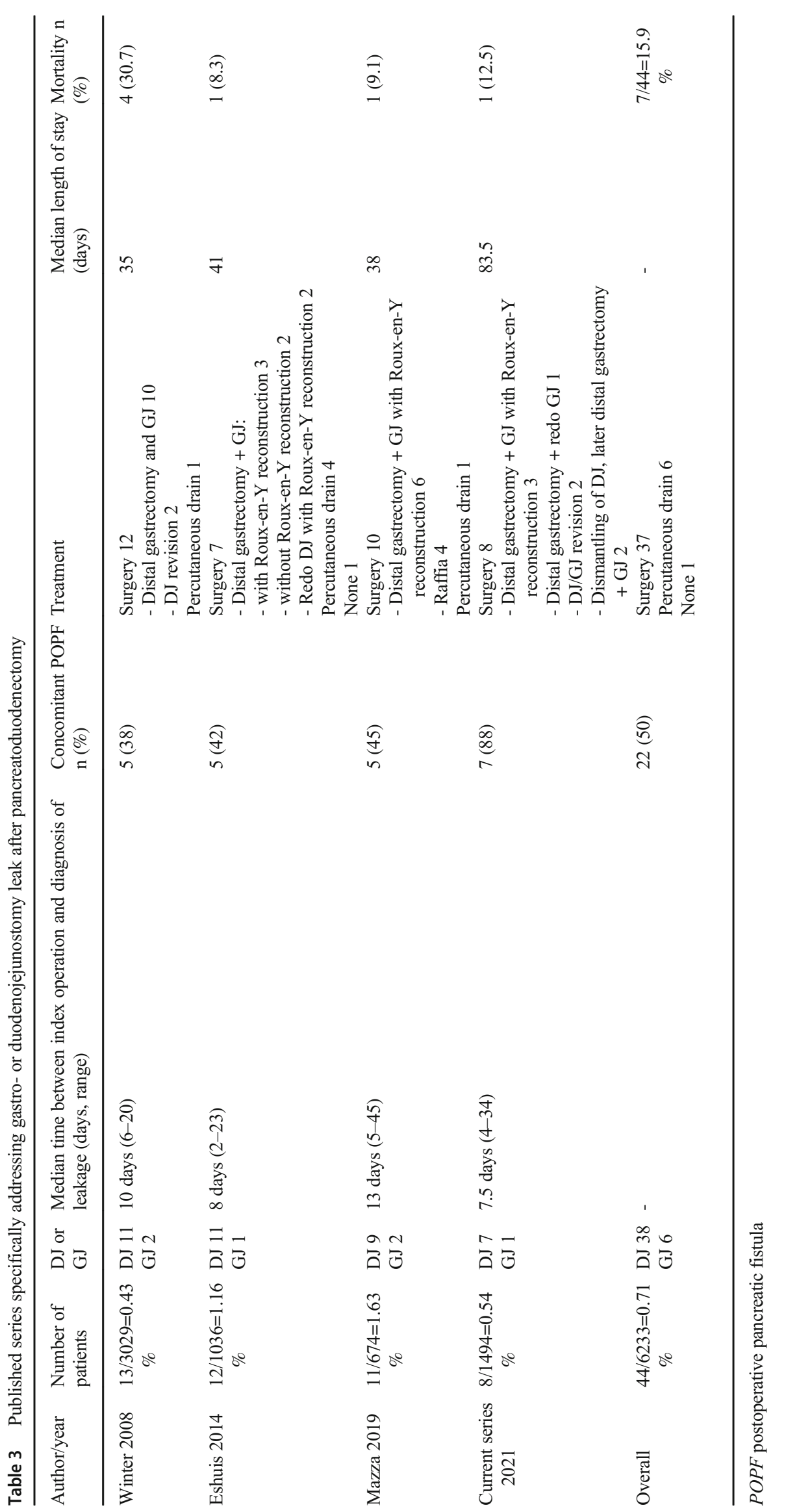


$=0.001)$ in patients with GJ leakage [4]. Because of the low number of events, multivariate analysis for the identification of possible risk factors for DJ/GJ leakage was not performed in the studies by Mazza and Eshuis. Although based on small study samples, patients developing GJ/DJ leak after PD seems to have undergone a more demanding PD with longer operative time and increased intraoperative blood loss.

A difference between DJ and GJ in the occurrence of a leak has not been reported. Huttner et al. performed a Cochrane review to compare the effectiveness of $\mathrm{CPD}$ and PPPD and identified eight randomized clinical trials with a total of 512 participants [2]. The review revealed no relevant differences in mortality, morbidity, and survival between the two operations, and no analysis or comments on the GJ/DJ leak rate was made in that review. A search of the individual eight papers revealed only five reported cases of GJ/DJ leak, in the largest series by Tran et al. of 170 consecutive patients $(n=2)$ and in a smaller series of 27 patients by Srinarmwong $(n=3)[1,11]$. Moreover, in a systematic review and meta-analysis, Hajibandeh et al. found no significant difference in the incidence of anastomotic leak between stapled anastomosis (SA) versus hand-sewn anastomosis of GJ or DJ in PD [12].

In the current study, all patients underwent a relaparotomy for definitive treatment of the leak, although a conservative approach was initially attempted in one patient. Eshuis et al. reported four cases successfully treated with percutaneous drainage alone, whereas Winter and Mazza treated one patient each with percutaneous drainage alone [3-5]. Overall, surgery was the treatment of choice in $84 \%$ of the patients (Table 3), and in all studies, the authors emphasized the importance of prompt diagnosis and operative intervention for a good outcome. Review of the literature revealed only one successful attempt with endoscopic treatment for this complication. Honig et al. reported advanced endoscopic rescue of a DJ leak after a PPPD in a post-esophagectomy patient with pancreatic cancer [13]. However, the role of endoscopic treatment for this rare complication after PD remains to be established.

Under salvage conditions dismantling the GJ/DJ anastomosis completely, closing the pyloric/gastric and jejunal end, performing a catheter gastrostomy, and feeding jejunostomy may be a bail-out strategy (Fig. 1). Given the rare incidence of GJ/DJ leak after PD, this salvage procedure has been scarcely reported in the literature [14]. However, for esophagogastric resections, it is well described that the anastomosis sometimes has to be taken down in case of fulminant sepsis or large defects. Diversion then needs to be taken into consideration, with restoration of intestinal continuity at a later date [15]. In the two patients in the current study, intestinal continuity could be restored and a GJ was successfully performed approximately 6 months after the dismantling procedure. In the authors' personal experience, at least 6-month time is necessary to allow healing of the concomitant POPF, resolution of the inflammatory changes, and restitution of nutrition.

\section{Conclusion}

GJ/DJ leak is a rare complication after PD. Prompt diagnosis and early intervention is important, and in most cases, a surgical intervention is necessary for a good outcome. Under salvage conditions, dismantling of the GJ/DJ anastomosis may be necessary. The role of interventional radiological or endoscopic procedures in the management of this complication remains to be established.

Acknowledgements We thank Dr. Uzma Ilyas who kindly made the illustration.

Funding Open access funding provided by University of Oslo (incl Oslo University Hospital). KJL received a research grant from South-Eastern Norway Regional Health Authority (2019029).

\section{Declarations}

Conflict of Interest The authors declare no competing interests.

Open Access This article is licensed under a Creative Commons Attribution 4.0 International License, which permits use, sharing, adaptation, distribution and reproduction in any medium or format, as long as you give appropriate credit to the original author(s) and the source, provide a link to the Creative Commons licence, and indicate if changes were made. The images or other third party material in this article are included in the article's Creative Commons licence, unless indicated otherwise in a credit line to the material. If material is not included in the article's Creative Commons licence and your intended use is not permitted by statutory regulation or exceeds the permitted use, you will need to obtain permission directly from the copyright holder. To view a copy of this licence, visit http://creativecommons.org/licenses/by/4.0/.

\section{References}

1. Tran KT, Smeenk HG, van Eijck CH, Kazemier G, Hop WC, Greve JW et al. Pylorus preserving pancreaticoduodenectomy versus standard Whipple procedure: a prospective, randomized, multicenter analysis of 170 patients with pancreatic and periampullary tumors. Ann Surg. 2004;240(5):738-45.

2. Huttner FJ, Fitzmaurice C, Schwarzer G, Seiler CM, Antes G, Buchler MW et al. Pylorus-preserving pancreaticoduodenectomy (pp Whipple) versus pancreaticoduodenectomy (classic Whipple) for surgical treatment of periampullary and pancreatic carcinoma. Cochrane Database Syst Rev. 2016;2:CD006053.

3. Winter JM, Cameron JL, Yeo CJ, Lillemoe KD, Campbell KA, Schulick RD. Duodenojejunostomy leaks after pancreaticoduodenectomy. J Gastrointest Surg. 2008;12(2):263-9.

4. Eshuis WJ, Tol JA, Nio CY, Busch OR, van Gulik TM, Gouma DJ. Leakage of the gastroenteric anastomosis after pancreatoduodenectomy. Surgery. 2014;156(1):75-82.

5. Mazza M, Crippa S, Pecorelli N, Tamburino D, Partelli S, Castoldi $\mathrm{R}$ et al. Duodeno-jejunal or gastro-enteric leakage after pancreatic resection: a case-control study. Updates Surg. 2019;71(2):295-303.

6. Kleive D, Sahakyan MA, Berstad AE, Verbeke CS, Gladhaug IP, Edwin B et al. Trends in indications, complications and outcomes 
for venous resection during pancreatoduodenectomy. Br J Surg. 2017;104(11):1558-67.

7. Kleive D, Sahakyan MA, Labori KJ, Lassen K. Nasogastric Tube on Demand is Rarely Necessary After Pancreatoduodenectomy Within an Enhanced Recovery Pathway. World J Surg. 2019;43(10):2616-22.

8. Bassi C, Marchegiani G, Dervenis C, Sarr M, Abu Hilal M, Adham $\mathrm{M}$ et al. The 2016 update of the International Study Group (ISGPS) definition and grading of postoperative pancreatic fistula: 11 Years After. Surgery. 2017;161(3):584-91.

9. Wente MN, Veit JA, Bassi C, Dervenis C, Fingerhut A, Gouma DJ et al. Postpancreatectomy hemorrhage $(\mathrm{PPH})$ : an International Study Group of Pancreatic Surgery (ISGPS) definition. Surgery. 2007;142(1):20-5.

10. Koch M, Garden OJ, Padbury R, Rahbari NN, Adam R, Capussotti $\mathrm{L}$ et al. Bile leakage after hepatobiliary and pancreatic surgery: a definition and grading of severity by the International Study Group of Liver Surgery. Surgery. 2011;149(5):680-8.

11. Srinarmwong C, Luechakiettisak P, Prasitvilai W. Standard whipple's operation versus pylorus preserving pancreaticoduodenectomy: a randomized controlled trial study. J Med Assoc Thai. 2008;91(5):693-8.
12. Hajibandeh S, Hajibandeh S, Khan RMA, Malik S, Mansour M, Kausar A et al. Stapled anastomosis versus hand-sewn anastomosis of gastro/duodenojejunostomy in pancreaticoduodenectomy: A systematic review and meta-analysis. Int J Surg. 2017;48:1-8.

13. Honig SE, Lundgren MP, Kowalski TE, Lavu H, Yeo CJ. Advanced Endoscopic Rescue of a Complication (Duodenojejunostomy Leak) After a Pylorus-Preserving Pancreaticoduodenectomy in a Post-Esophagectomy Patient with Pancreatic Adenocarcinoma: A Case Report and Review of the Literature. J Pancreat Cancer. 2020;6(1):5-11.

14. Reddy JR, Saxena R, Singh RK, Pottakkat B, Prakash A, Behari A et al. Reoperation following Pancreaticoduodenectomy. Int J Surg Oncol. 2012;2012:218248.

15. Hummel R, Bausch D. Anastomotic Leakage after Upper Gastrointestinal Surgery: Surgical Treatment. Visc Med. 2017;33(3):207-11.

Publisher's Note Springer Nature remains neutral with regard to jurisdictional claims in published maps and institutional affiliations. 